\title{
émulations
}

\section{Jasper van der Steen - Memory Wars in the Low Countries, 1566-1700}

\section{Julien Régibeau}

Émulations - Revue de sciences sociales

2017, «Comptes rendus critiques, En ligne »

\section{Article disponible à l'adresse suivante}

https://ojs.uclouvain.be/index.php/emulations/article/view/7303

\section{Pour citer cet article}

Julien Régibeau, «Jasper van der Steen — Memory Wars in the Low Countries, 1566$1700 »$, Émulations, en ligne. Mise en ligne le 9 janvier 2017.

DOI : 10.14428/emulations.cr.031

Distribution électronique : Université catholique de Louvain (Belgique) : ojs.uclouvain.be

(C) Cet article est mis à disposition selon les termes de la Licence Creative Commons Attribution, Pas d'Utilisation Commerciale 4.0 International. http://creativecommons.org/licenses/by-nc/4.0/

Éditeur : Émulations - Revue de sciences sociales / Presses universitaires de Louvain https://ojs.uclouvain.be/index.php/emulations

ISSN électronique : 1784-5734

UCL PRESSES

UNIVERSITAIRES 


\title{
Jasper van der Steen - Memory Wars in the Low Countries, 1566-1700
}

\author{
Julien Régibeau ${ }^{1}$
}

Recensé : Jasper van der Steen, Memory Wars in the Low Countries, 1566-1700, Leyde, Brill (Studies in Medieval and Reformation Traditions, 190), 2015, 370 p.

$\mathrm{Au}$ XVII siècle, auraient émergé, dans les Pays-Bas et dans les Provinces-Unies, deux mémoires de la Révolte (1566-1581) radicalement différentes. Partant de ce constat, l'historien Jasper van der Steen construit son ouvrage — fruit d'une thèse défendue en 2014 sous l'égide de la professeure Judith Pollmann - autour d'une triple problématique : comment et pourquoi apparaissent au Nord et au Sud des interprétations si conflictuelles de la Révolte? Pourquoi, ensuite, celles-ci restent-elles pertinentes aussi longtemps ? Quel rôle enfin jouèrent les mémoires publiques de la Révolte dans la formation identitaire des Pays-Bas et des Provinces-Unies?

Il a fallu attendre les années 80 et 90 pour que se développe une historiographie des pratiques mémorielles sensible à l'étude de l'identité nationale et de la mémoire culturelle (Anderson, 1983 ; Hobsbawm, 1983 ; Groenveld, 1980 ; Nora, 1992 ; Duke, 2004). S'inscrivant dans le sillage de ce renouveau, l'auteur choisit d'appliquer les problématiques de l'histoire culturelle de la mémoire au XVII e siècle, époque trop souvent négligée par une recherche qui envisage la construction nationale et les rapports publics au passé qu'elle suppose comme un fait contemporain (Lorenz, 2010). Pour cela, Jasper van der Steen choisit de réaliser une étude comparée des politiques mémorielles des PaysBas et des Provinces Unies. Se basant sur le concept de mémoire - entendue comme l'interaction dynamique entre une communauté et son passé - proposé par Astrid Erll (2008), l'auteur forge, pour servir son propos, le terme de mémoire de guerre, qui traduit le fait que toute lutte politique suppose une interprétation et un usage conflictuels du passé.

Dans le premier chapitre de son livre ("Memory in the Making : The First Decades», p. 31-62), l'auteur part des prémices de la Révolte afin de pister les usages du passé faits par le gouvernement de Bruxelles et les rebelles dans le but de décrédibiliser l'action de leur adversaire. À la veille des troubles, les états de Brabant brandissent les privilèges de la Joyeuse Entrée afin de s'opposer à la réorganisation des diocèses et à la persécution religieuse. Face à ces démarches, Granvelle mobilise la mémoire plus récente des troubles auxquels Charles V s'est opposé pour, au contraire, porter Philippe II à agir

${ }^{1}$ Aspirant F.R.S.-FNRS, doctorant en Histoire des Temps Modernes, Unité de recherches Transitions, Moyen Âge \& première Modernité, Université de Liège. 
énergiquement. L'éclatement de la Révolte crée ensuite une rupture entre l'action des rebelles et l'invocation du passé. Cette tension mène les Habsbourg à leur reprocher d'être des faiseurs de troubles et de nouveautés. Face à cela, les révoltés établissent une mémoire des événements récents qui tend à prouver la justesse de leur cause. Dans ce contexte, l'action de Guillaume d'Orange est originale. Désirant aplanir toute dissension interne, celui-ci évite les polémiques religieuses pour se concentrer sur la cruauté dont feraient preuve les soldats espagnols et l'inquisition. Dans le Sud, la reconquête d'Alexandre Farnèse met cependant fin à toute commémoration publique de la Révolte. Le gouverneur-général diffuse l'idée que les troubles ont été une interruption momentanée de la continuité dynastique. Dans les Provinces du Nord, par contre, la mémoire de la Révolte survit mais elle demeure peu structurée par les autorités centrales. Seuls les écrits de Guillaume d'Orange et de certains étrangers comme Guichardin donnent des événements l'interprétation d'un conflit général des Pays-Bas contre le pouvoir espagnol. Au Nord comme au Sud, enfin, il faut attendre le début du XVII siècle pour que les premiers ouvrages historiographiques sur les troubles soient publiés.

Dans le deuxième chapitre (« Two Historical Canons », p. 63-107), l'auteur étudie comment deux conceptions politiques opposées mènent à l'élaboration de deux canons différents du passé. Dans les Provinces-Unies, les négociations en vue de la trêve de douze ans entrainent la mise en place d'une lecture unifiée de la Révolte. Ce sont surtout les factions anti-paix qui contribuent à la création de ce récit identitaire. Elles proposent une mémoire déconfessionnalisée et populaire de la Révolte, basée, comme le récit de Guillaume d'Orange, sur une logique inclusive qui dessine l'image du hollandais innocent face à l'espagnol cruel et pervers. Dans les Pays-Bas, par contre, la politique de l'oubli est encore privilégiée sous les Archiducs. Elle est facilitée par le recours régulier des autorités à un vaste réservoir historique précédant les événements de la Révolte. Mais l'amnésie ne peut être la seule posture pour des générations qui ont vécu personnellement les troubles et qui soutiennent l'effort de guerre au quotidien. Pour faire face à cette discordance, les autorités ne manquent pas de célébrer des événements récents qui glorifient la maison d'Autriche. La dévotion qui en découle fonde le catholicisme en vérité immémoriale. Elle tend du même coup à faire de la rébellion un accident de l'histoire et du calvinisme une hérésie nouvelle et cruelle. Enfin, des interactions mémorielles entre le Nord et le Sud se font jour, des disputes entre des auteurs des deux territoires ayant notamment lieu autour de la bonne lecture à donner au passé de la Révolte.

Le troisième chapitre (" Dynastic Identity and the Revolt », p. 108-140) a pour but de montrer comment, au début du XVII ${ }^{\mathrm{e}}$ siècle, la maison d'Autriche et la maison d'Orange-Nassau travaillent la mémoire publique pour imposer le récit de la continuité dynastique de leur famille et comment, en poursuivant cet objectif, elles composent avec le passé de la Révolte. Dans le Sud, la cession des Pays-Bas aux Archiducs est replacée dans la continuité dynastique habsbourgeoise. L’idéologie de la Pietas Austriaca 
participe de ce mouvement en faisant d'Albert et d'Isabelle les défenseurs de la chrétienté au même titre que leurs prédécesseurs. Cette piété sert ensuite de modèle à la haute noblesse et contribue donc à homogénéiser la mémoire. Dans les ProvincesUnies, par contre, c'est l'action des membres de la famille d'Orange durant la Révolte qui fonde le prestige de la dynastie. Très rapidement, Guillaume attache en effet son destin à celui des Pays-Bas qui combattent l'Espagne. À la fin du xvie siècle, sa mémoire est peu à peu glorifiée par les autorités alors que la réputation des succès militaires de son fils, Maurice, est diffusée à travers les Provinces. La survenue de la trêve de douze ans et la mort, en 1618, de Philippe-Guillaume marquent un tournant favorable pour le développement de l'autorité de Maurice. À cette époque est bâti un imaginaire politique basé sur le triptyque « Dieu-la République-Orange ».

Dans le quatrième chapitre (" A Contested Past », p. 141-178), l'auteur analyse de quelle manière la mémoire de la Révolte est employée, dès la première moitié du XVII siècle, comme une arme polémique au service de querelles politiques et religieuses internes. Le phénomène est étudié à travers la querelle autour de l'arminianisme, durant les années 1610, et la conspiration des malcontents contre l'autorité habsbourgeoise, en 1632. Dans les Provinces-Unies, la dispute qui oppose « remonstrants » et «contreremonstrants » mobilise la mémoire publique de la Révolte et tend à confessionnaliser celle-ci. Le conflit conduit à une querelle des origines : alors que les contreremonstrants accusent leurs adversaires de nouveauté et en appellent au respect de l'union d'Utrecht ou de la Confessio Belgica, les tenants de l'arminianisme leur opposent l'antiquité de leur pensée. Dans les deux camps, la mémoire de Guillaume d'Orange est brandie, chacun se considérant comme l'héritier de son action. Dans le Sud, l'invasion des Pays-Bas espagnols par Frédéric-Henri d’Orange planifiée par Warfusée et Van der Bergh, en 1632, mène ce dernier à envoyer une lettre ouverte à l'archiduchesse Isabelle. Dans ce courrier, il s'érige en défenseur des intérêts du pays et compare la situation à la période de troubles qui suit la mort de Requesens. Afin de minimiser l'influence de ses adversaires, le gouvernement de Bruxelles, quant à lui, favorise la publication de La Flandre Fidelle, ouvrage qui s'appuie aussi sur les événements du xvie siècle, mais cette fois pour mettre en avant les vertus de l'Espagne. Suite à l'échec de la conspiration, Isabelle écrit une lettre au roi Philippe IV dans laquelle elle justifie la victoire par ses propres choix politiques, élaborés en ayant en mémoire les erreurs du passé.

À la reprise des hostilités, en 1621, le conflit a changé de nature. Bien que les générations qui ont vécu les premiers troubles disparaissent, l'usage du passé de la Révolte demeure vivace. Dans le cinquième chapitre («Stakeholders », p. 179-215), l'auteur se demande pourquoi cette mémoire conserve sa puissance politique. Ce phénomène est notamment dû à plusieurs familles de la haute noblesse qui s'appuient sur les actions de leurs membres durant les troubles pour laver leur honneur et revendiquer un bien, un privilège ou de l'autorité. Dans les Pays-Bas espagnols, plus spécifiquement, le passage des générations rend l'interprétation des événements plus souple et davantage 
fondée sur l'idée d'une lutte entre deux états distincts. Par ailleurs, de nouvelles pratiques mémorielles, moins confessionnalisées, émergent. Afin de mobiliser toutes les sensibilités autour du projet de résistance, plusieurs œuvres cherchent à victimiser le groupe dans son entièreté face aux atrocités des armées des Provinces-Unies. Au Nord, par contre, ce sont les débats entre pro - et anti-paix qui continuent à structurer l'usage public du passé de la Révolte jusqu'en 1648. Le parti qui s'oppose à la paix puise dans les exempla fournis par les troubles pour démontrer la duplicité des Espagnols et justifier la continuation des combats. En conséquence, après 1621, la Révolte contribue à développer deux sociétés militantes qui accordent une grande importance à la culture du conflit.

Dans le sixième chapitre (" Memories after Westphalia », p. 216-255), l'auteur poursuit chronologiquement son exploration de l'usage mémoriel de la Révolte en s'intéressant à la seconde moitié du XVII e siècle, époque où les Pays-Bas et les Provinces-Unies sont amenés à lutter ensemble contre la France. Il démontre qu'une nouvelle situation politique ne suppose pas la chute des anciens canons mémoriels mais plutôt leur redéploiement. La paix de Westphalie ne met pas fin immédiatement aux dissensions, religieuses notamment, entre le Sud et le Nord. Dans les Pays-Bas, une interprétation de la Révolte fustigeant les rebelles reste prégnante mais l'usage public de ce passé est rare. Au cours de la période, seuls l'absentéisme récurrent de la famille habsbourgeoise et les guerres de Louis xiv entrainent les autorités de Bruxelles à faire une utilisation politique de cette mémoire. Dans un cas comme l'autre le gouvernement est amené à mettre en avant la supériorité du modèle politique habsbourgeois face à la Raison d'État de la France et des Provinces-Unies. Au Nord, par contre, la Révolte est devenu un pilier de l'identité nationale et est considérée comme un véritable réservoir de références au service de la lutte politique. Durant la première période sans Stathouder (1650-1672), le parti orangiste rappelle le rôle de Guillaume $\mathrm{i}^{\text {er }}$ lors des troubles afin de démontrer la nécessité du retour du Stathoudérat. Pour faire face à l'invocation de cette figure mythifiée, le parti des états est entrainé à défier le canon mémoriel qui lie le destin de la famille d'Orange-Nassau à l'identité des Provinces.

Dans le dernier chapitre de son livre ("Remediating the War », p. 256-285), l'auteur étudie comment la mémoire publique de la Révolte demeure vive et utile à une époque où la population perçoit de plus en plus l'éloignement temporel de ces événements. À partir de la fin du XVII siècle, il est difficile de trouver des réemplois originaux de ce passé. Les deux narrations dominantes se sont stabilisées. Leur usage politique ne semble plus porter des enjeux aussi importants qu'auparavant. Pour autant, cela ne signifie pas que d'autres références historiques auraient remplacé l'intelligibilité de cette mémoire. Les cérémonies des centenaires montrent qu'aux Pays-Bas, la Révolte, souvent replacée dans des chronologies plus vastes (religieuses, dynastiques...), contribue à définir l'identité du "vrai habitant des Pays-Bas », fondée sur la loyauté envers les Habsbourg et l'orthodoxie religieuse. Dans les Provinces-Unies, par contre, les nouvelles guerres menées contre des états étrangers conduisent les disputes mémorielles 
internes des années 1650-1672 à se fondre dans une nouvelle approche consensuelle de la Révolte.

L'ouvrage est bâti d'une part sur une bibliographie consistante et actualisée qui puise essentiellement dans la littérature anglo-saxonne et néerlandaise et, d'autre part, sur l'analyse d'un ample corpus de sources diverses. On pourra regretter une lecture des documents qui laisse peu de place à l'intelligibilité du contexte de production de chacune de ces sources, lieu pourtant privilégié du rôle performatif de l'intentionnalité. On déplorera également le grand écart qu'il y a entre les pratiques mémorielles étudiées et les mots chargés d'en rendre compte ; les concepts employés au fil de l'analyse découlant plus du sens qu'ils revêtent aujourd'hui dans l'usage académique ou courant que de celui qu'ils revêtent dans les documents du passé. On pointera enfin le caractère fonctionnaliste d'une étude qui présuppose que la construction d'une mémoire publique est la conséquence logique de stratégies politiques conscientes ; une étude qui nie de ce fait tout rôle au hasard, à l'incertitude et à l'irrationalité des acteurs dans la construction de l'histoire, une étude qui néglige surtout la tension herméneutique qui sépare l’intention, l’action et le résultat obtenu (Bourdieu, 1972 ; Certeau, 1980 ; Ricœur, 1988 ; Lepetit, 1995 ; Chartier, 1996).

Ces réserves n'entachent cependant pas à nos yeux les qualités de cet ouvrage. Sérieuse, rigoureuse et bien réalisée, l'étude de Jasper van der Steen nous donne à voir une vaste analyse comparée de l'usage de la mémoire de la Révolte sur plus d'un siècle et demi d'histoire. Les conclusions rappelant que la construction identitaire d'un état à travers l'appropriation d'un passé recomposé n'est pas le seul fruit de la période contemporaine mais mérite au contraire d'être appréhendée dans ses temps et ses modalités multiples sont particulièrement stimulantes.

\section{Bibliographie}

Bourdieu P. (1972), Esquisse d'une théorie de la pratique. Précédé de trois études d'ethnologie kabyle, Genève, Droz.

CERTEAU M. de (1990 [1980]), L'Invention du quotidien, t. 1, Arts de faire, Paris, Gallimard (« Folio essais »).

CHARTIER R. (1998), Au bord de la falaise : l'histoire entre certitudes et inquiétude, Paris, A. Michel («Bibliothèque Albin Michel de l’histoire»).

DuKE A. (2004), «The Elusive Netherlands : the Question of National Identity in the Early Modern Low Countries on the Eve of the Revolt», in Bijdragen en mededelingen betreffende de geschiedenis der Nederlanden, vol 119, $\mathrm{n}^{\circ} 1, \mathrm{p} .10-38$.

ERLL A. (2008), «Cultural Memory Studies : An Introduction », in A. ERLL, A. NüNNING, Cultural Memory Studies : An International and Interdisciplinary Handbook, New York, Walter de Gruyter (« Media and cultural memory »), p. 1-11. 
GROENVELD S. (1980), « Natie en nationaal gevoel in de zestiende-eeuwse Nederlanden », in Nederlands Archievenblad, vol. 84, n 3, p. 372-387.

HoBSBAwM E. (2000 [1983]), « Introduction : Inventing Traditions », in E. Hobsbawm, T. Ranger, The Invention of Tradition. Cambridge, Cambridge University Press (Past and present publications).

LEPETIT B. (1995), Les formes de l'expérience : une autre histoire sociale, Paris, A. Michel.

Lorenz Ch. (2010), «Unstuck in Time. Or: The Sudden Presence of the Past », in K. Tilmans, F. van Vree, J. Winter, Performing the Past: Memory, History and Identity in Modern Europe, Amsterdam, Amsterdam University Press, p. 67-102.

NORA P. (2000 [1983]), « Comment écrire l’histoire de France ? », in Les lieux de mémoire, t. 3, Les France, 1 ère partie, Conflits et partages, Paris, Gallimard («Bibliothèque illustrée des histoires »), p. 11-32.

RICCEUR P. (1988), « L’identité narrative », in Esprit, vol. 140/141, n 7/8, p. 295-304. 